\title{
Spacetime Structures of Quantum Particles
}

\author{
Vu B Ho ${ }^{*}$ \\ Advanced Study, 9 Adela Court, Mulgrave, Victoria 3170, Australia \\ *Corresponding author: vubho@bigpond.net.au
}

\begin{abstract}
In this work first we show that the three main formulations of physics, namely, Newton's second law of motion, Maxwell field equations of electromagnetism and Einstein field equations of gravitation can be formulated in similar covariant forms so that the formulations differ only by the nature of the geometrical objects that represent the corresponding physical entities. We show that Newton's law can be represented by a scalar, the electromagnetic field by a symmetric affine connection or a dual vector, and the gravitational field by a symmetric metric tensor. Then with the covariant formulation for the gravitational field we can derive differential equations that can be used to construct the spacetime structures for short-lived and stable quantum particles. We show that geometric objects, such as the Ricci scalare curvature and Gaussian curvature, exhibit probabilistic characteristics. In particular, we also show that Schrödinger wavefunctions can be used to construct spacetime structures for the quantum states of a quantum system, such as the hydrogen atom. Even though our discussions in this work are focused on the microscopic objects, the results obtained can be applied equally to the macroscopic phenomena.
\end{abstract}

Keywords: spacetime structures, quantum particles, general relativity, schrödinger wavefunctions, covariant formulations

Cite This Article: Vu B Ho, "Spacetime Structures of Quantum Particles." International Journal of Physics, vol. 6, no. 4 (2018): 105-115. doi: 10.12691/ijp-6-4-2.

\section{Introduction}

In classical physics, particle is a general term that refers to a localized object that can be used to model physical theories. The motion of a particle can be described in a deterministic and predicable pattern within the framework of Newtonian mechanics. In quantum physics, the concept of the classical particle is still retained even though in the realm of quantum mechanics a quantum particle exhibits the phenomenon of matter wave, which, if not being simply probabilistically interpreted, is a physical occurrence due to an extended medium rather than a single physical object occupying a single position at a particular time in space. This epistemological problem raises the question of whether the concept of particle as a localized object is adequate for modeling physical theories or in fact physical theories should be based on the model of a particle as an extended object. In this work we show that quantum particles that are formed from the microscopic spacetime structures can be regarded as elementary particles in three-dimensional Euclidean space and can be assumed to possess the geometric and topological structure of a differentiable manifold, and a physical theory can be formulated as the dynamics of its spacetime structures. An intrinsic geometric dynamics of a differentiable manifold is a geometric process of evolution that can be described by physical theories such as general relativity [1] or geometrical theories such as the Ricci flow $[2,3]$. Even though we will not represent them in this work, for a more complete description an elementary particle should be represented as a CW complex $[4,5,6]$. In this work we will deal with the following topics. In Section 2 we show that classical physics that includes Newtonian mechanics, Maxwell field equations of electromagnetism and Einstein field equations of general relativity can be formulated in a general covariant form. In Section 3 we show that geometric objects such as the Ricci scalar curvature also have a probabilistic character. In Section 4 we show that Schrödinger wavefunctions can be used as mathematical objects to construct intrinsic geometric spacetime structures of quantum particles.

\section{A Covariant Formulation of Classical Physics}

In this section we show that the three main formulations of physics, namely, Newton's second law of motion, the field equations of the electromagnetic field and the field equations of the gravitational field can be formulated in similar covariant forms so that the formulations differ only by the nature of the geometrical objects that represent the corresponding physical entities. Fundamentally, Newton's law can be represented by a scalar, the electromagnetic field by a dual vector or by a symmetric affine connection, and the gravitational field by a symmetric metric tensor. In classical physics, for conservative forces, Newton's second law can be written in terms of a potential energy $V$ as [7]

$$
\begin{aligned}
& \mathbf{F}=m \frac{d^{2} \mathbf{r}}{d t^{2}} \\
& \mathbf{F}=-\nabla V .
\end{aligned}
$$


Maxwell field equations of the electromagnetic field can be written as $[8,9]$

$$
\begin{gathered}
\nabla . \mathbf{j}_{e}+\frac{\partial \rho_{e}}{\partial t}=0 \\
\nabla . \mathbf{E}=\frac{\rho_{e}}{\epsilon} \\
\nabla . \mathbf{B}=0 \\
\nabla \times \mathbf{E}+\frac{\partial \mathbf{B}}{\partial t}=0 \\
\nabla \times \mathbf{B}-\epsilon \mu \frac{\partial \mathbf{E}}{\partial t}=\mu \mathbf{j}_{e} .
\end{gathered}
$$

And Einstein field equations of the gravitational field can be written in the following tensorial form [1]

$$
R_{\alpha \beta}-\frac{1}{2} g_{\alpha \beta} R=\kappa T_{\alpha \beta}
$$

The three main systems of dynamical equations of physics have different mathematical formulations because on one hand, Newton's second law and Maxwell field equations were basically derived from physical laws that had been established from experiments therefore they contain experimentally defined physical entities. On the other hand, Einstein field equations were proposed purely from scientific and mathematical reasoning. However, in the following we will show that all three dynamical systems can be formulated in the same general covariant form and they can be represented by the following general equation

$$
\nabla_{\beta} M=k J
$$

where $M$ is a mathematical object that represents the corresponding physical system and $\nabla_{\beta}$ is a covariant derivative. For Newton's second law $M=E$ and $J=0$. For Maxwell field equations of electromagnetism $M=F^{\alpha \beta}$ and $J$ can be identified with the electric and magnetic currents. And for the field equations of general relativity $M=R^{\alpha \beta}$ and $J$ can be defined in terms of a metric and Ricci scalar curvature.

In fact, Maxwell field equations of the electromagnetic field have already been formulated in the following covariant form

$$
\frac{\partial F^{\alpha \beta}}{\partial x^{\alpha}}=\mu j^{\beta}
$$

where the electromagnetic tensor $F^{\alpha \beta}$ is expressed in terms of the four-vector potential $A^{\mu} \equiv(V, \mathbf{A})$ as $F^{\mu v} \equiv$ $\partial^{\mu} A^{v}-\partial^{v} A^{\mu}$. The four-current $j^{\mu}$ is defined as $j^{\mu} \equiv\left(\rho_{e}, \mathbf{j}_{e}\right)$. Since the electromagnetic tensor $F^{\alpha \beta}$ is anti-symmetric, it can be expressed in terms of a dual vector. In terms of the electromagnetic tensor $F^{\alpha \beta}$, the electromagnetic energy-momentum tensor $T^{\alpha \beta}$ for the free electromagnetic field with the defined Lagrangian of the form $L=-\frac{1}{4} F^{\alpha \beta} F_{\alpha \beta}$ can be established as

$$
T^{\alpha \beta}=\epsilon_{0} c^{2}\left(\eta_{\mu v} F^{\alpha \mu} F^{v \beta}+\frac{1}{4} \eta^{\alpha \beta} F_{\lambda v} F^{\lambda v}\right)
$$

where $\eta_{\alpha \beta}$ is the Minkowski metric tensor $[8,9]$. We now show how a covariant form as given in Equation (10) for the electromagnetic field can be formulated for Newton's law of mechanical dynamics and the field equations of the gravitational field.

In order to formulate Newton's second law of dynamics covariantly, we write Newton's second law given in Equations (1) and (2) in terms of the potential energy $V$ with the coordinate notation $x^{\mu}=(x, y, z)$ as follows

$$
m \frac{d^{2} x^{\mu}}{d t^{2}}+\frac{\partial V}{\partial x^{\mu}}=0
$$

From the definition of work done in classical mechanics, which is defined as the line integral of a force $\mathbf{F}$ along a path $C, W=\int_{C} \mathbf{F} . d \mathbf{r}$, the conserved energy $E$ for a particle of inertial mass $m$ is established as

$$
E=\frac{1}{2} m \sum_{\mu=1}^{3}\left(\frac{d x^{\mu}}{d t}\right)^{2}+V .
$$

From Equation (13), we obtain the following relation by differentiation

$$
\frac{\partial E}{\partial x^{\mu}}=m \frac{d^{2} x^{\mu}}{d t^{2}}+\frac{\partial V}{\partial x^{\mu}} .
$$

If the dynamics of the particle satisfies Newton's second law given in Equation (12) then we obtain

$$
\frac{\partial E}{\partial x^{\mu}}=0 \text {. }
$$

It is seen that Equation (15) has the covariant form similar to Maxwell equations of the electromagnetic field given in Equation (10). However, the covariant equation for Newton's dynamics is related to a scalar rather than a tensor as in the field equations for electromagnetism. Furthermore, it is interesting to observe the following. While the total energy $E=T+V$ of a physical system is the sum of the kinetic energy and the potential energy, the Lagrangian $L=T-V$ of a physical system is essentially the difference between the kinetic energy and the potential energy. Similar to Equation (14), we can write

$$
\frac{\partial L}{\partial x^{\mu}}=m \frac{d^{2} x^{\mu}}{d t^{2}}-\frac{\partial V}{\partial x^{\mu}} .
$$

Equation (16) can be used to describe, for example, the expansion of a physical system in which the term $\partial V / \partial x^{\mu}$ can be considered as a repulsive force. As in the case of total energy $E$, if the Lagrangian $L$ of a physical system is conserved, i.e.,

$$
\frac{\partial L}{\partial x^{\mu}}=0
$$

then we obtain

$$
m \frac{d^{2} x^{\mu}}{d t^{2}}-\frac{\partial V}{\partial x^{\mu}}=0 .
$$

We now show that the field equations of the gravitational field can be proposed and formulated in a covariant form similar to the covariant form of the 
electromagnetic field given in Equation (10). It is shown in differential geometry that the Ricci tensor $R^{\alpha \beta}$ satisfies the Bianchi identities [10]

$$
\nabla_{\beta} R^{\alpha \beta}=\frac{1}{2} g^{\alpha \beta} \nabla_{\beta} R
$$

where $R=g^{\alpha \beta} R_{\alpha \beta}$ is the Ricci scalar curvature. Even though Equation (19) is purely geometrical, it has a covariant form similar to Equation (10) for the electromagnetic tensor $\partial_{\alpha} F^{\alpha \beta}=\mu j^{\beta}$ defined in Euclidean space. If the quantity $\frac{1}{2} g^{\alpha \beta} \nabla_{\beta} R$ can be identified as a physical entity, such as a four-current of gravitational matter, then Equation (19) has the status of a dynamical law of a physical theory. In this case a four-current $j^{\alpha}=\left(\rho, \mathbf{j}_{i}\right)$ can be defined purely geometrical as

$$
j^{\alpha}=\frac{1}{2} g^{\alpha \beta} \nabla_{\beta} R .
$$

In later sections we will show that the purely geometrical four-current $j^{\alpha}$ defined by Equation (20) can be established as physical entities, however, in the following we want to show that for the case of a purely gravitational field in which $\frac{1}{2} g^{\alpha \beta} \nabla_{\beta} R=0$, the proposed field equations given in Equation (19) also arrive at the same results as those from Einstein's formulation of the gravitational field. For a purely gravitational field, Equation (19) reduces to the equation

$$
\nabla_{\beta} R^{\alpha \beta}=0
$$

The field equations for the gravitational field given in Equation (21) play the role of Maxwell field equations for the free electromagnetic field. Even though rigorous solutions to the dynamical field equations given in Equation (21) would require laborious mathematical investigations, we can obtain solutions found from the original Einstein field equations, such as Schwarzschild solution, by observing that since $\nabla_{\mu} g^{\alpha \beta} \equiv 0$ Equation (21) implies

$$
R^{\alpha \beta}=\Lambda g^{\alpha \beta}
$$

where $\Lambda$ is an undetermined constant. Equation (22) can also be written in a covariant form as

$$
R_{\alpha \beta}=\Lambda g_{\alpha \beta} \text {. }
$$

Using the identities $g_{\alpha \beta} g^{\alpha \beta}=4$ and $g_{\alpha \beta} R^{\alpha \beta}=R$, we obtain $\Lambda=R / 4$. If we consider a centrally symmetric gravitational field with the metric

$$
d s^{2}=e^{\psi} c^{2} d t^{2}-e^{\chi} d r^{2}-r^{2}\left(d \theta^{2}+\sin ^{2} \theta d \phi^{2}\right)
$$

then the Schwarzschild solution can be found as [11]

$$
\begin{aligned}
d s^{2} & =\left(1-\frac{2 G M}{r}-\frac{\Lambda r^{2}}{3}\right) c^{2} d t^{2} \\
& -\left(1-\frac{2 G M}{r}-\frac{\Lambda r^{2}}{3}\right)^{-1} d r^{2}-r^{2}\left(d \theta^{2}+\sin ^{2} \theta d \phi^{2}\right)
\end{aligned}
$$

It is observed that, as in the case of the free electromagnetic field, the energy-momentum tensor $T_{\alpha \beta}$ for the gravitational field can be established if we define it through the relation $T_{\alpha \beta}=\frac{1}{\kappa}\left(R_{\alpha \beta}-\frac{1}{2} g_{\alpha \beta} R\right)$, which is Einstein field equations given in Equation (8). Furthermore, from Equation (23) we also obtain the relation $T_{\alpha \beta}=(\Lambda / \kappa) g_{\alpha \beta}$. It is interesting to mention here that, as shown in Appendix 1, the intrinsic geometric Ricci flow that was introduced by Hamilton can also be derived from Equation (21) and given as follows

$$
\frac{\partial g_{\alpha \beta}}{\partial t}=-2 R_{\alpha \beta} \text {. }
$$

Mathematically, the Ricci flow is a geometric process that can be employed to smooth out irregularities of a Riemannian manifold [2,3].

\section{Probabilistic Characteristics of Geometric Objects}

In this section, we discuss the possibility of identifying geometric objects with physical entities and we show that the identifications provide a route to formulate dynamical equations that describe probabilistic processes in physical theories. In particular, by assuming the purely geometrical Bianchi identities as covariant field equations of the gravitational field we are able to derive a geometric diffusion equation and a Schrödinger-like wave equation that can be used to describe random movement of particles as spacetime structures. First we want to show that the probabilistic characteristics of geometric objects also manifest even in semi-classical theory such as Bohr's theory of the hydrogen atom [12]. As shown in Appendix 2 , the momentum $p$ of the particle and the curvature $\kappa$ of its trajectory in a plane are related by the relation $p=\hbar \kappa$. According to the canonical formulation of classical physics, the particle dynamics is governed by the action principle $\delta S=\delta \int p d s=0$. Using the relation $p=\hbar \kappa$ and the expression of the curvature of a trajectory $f(x)$ in a plane, $\kappa=f^{\prime \prime} /\left(1+f^{\prime 2}\right)^{3 / 2}$, the action integral $S$ takes the form

$$
S=\int \hbar \kappa d s=\int \frac{\hbar f^{\prime \prime}}{1+f^{\prime 2}} d x
$$

It is shown in the calculus of variations that to extremise the integral $S=\int L\left(f, f^{\prime}, f^{\prime \prime}, x\right) d x$, the function $f(x)$ must satisfy the differential equation [13]

$$
\frac{\partial L}{\partial f}-\frac{d}{d x} \frac{\partial L}{\partial f^{\prime}}+\frac{d^{2}}{d x^{2}} \frac{\partial L}{\partial f^{\prime \prime}}=0 .
$$

However, with the functional of the form given in Equation (27), $L=\hbar f^{\prime \prime} /\left(1+f^{\prime 2}\right)$, it is straightforward to verify that the differential equation (28) is satisfied by any function $f(x)$. This result may be considered as a foundation for the Feynman's path integral formulation of quantum mechanics, which uses all classical trajectories of a particle in order to calculate the transition amplitude of a 
quantum mechanical system [14,15]. Since any path can be taken by a particle moving in a plane, if the orbits of the particle are closed, it is possible to represent each class of paths of the fundamental homotopy group of the particle by a circular path, since topologically, any path in the same equivalence class can be deformed continuously into a circular path. This validates Bohr's assumption of circular motion for the electron in a hydrogen-like atom. This assumption then leads immediately to the Bohr quantum condition

$$
\oint p d s=\hbar \oint \kappa d s=\hbar \oint \frac{d s}{r}=\hbar \oint d \theta=n h .
$$

The Bohr quantum condition possesses a topological character in the sense that the principal quantum number $n$ is identified with the winding number, which is used to represent the fundamental homotopy group of paths of the electron in the hydrogen atom.

Now we show that the geometric objects that are identified as physical entities from the covariant form of the field equations of the gravitational field given in Equation (19) also exhibit probabilistic characteristics. From the four-current of matter given in Equation (20), by letting $\alpha=0$, we obtain the matter density component of the four-current as

$$
\rho=j^{0}=\frac{1}{2} g^{0 \beta} \nabla_{\beta} R=\frac{1}{2} g^{0 \beta} \partial_{\beta} R .
$$

It is seen from Equation (30) that in order to be able to define matter in terms of geometric objects, the Ricci scalar must depend explicitly on the coordinates $x^{\mu}$. In particular, if the metric tensor $g^{\alpha \beta}$ is diagonal and the Ricci tensor depends explicitly on the temporal coordinate $x^{0}=c t$ then we have the geometrical density

$$
\rho=\frac{1}{2 c} g^{00} \partial_{t} R
$$

In order to give the mathematical entity $\rho$ a physical content, we introduce a dimensional constant $k_{1}$ and Equation (31) is rewritten as

$$
\rho=\frac{k_{1}}{2 c} g^{00} \partial_{t} R
$$

We will assume that the field equations of general relativity given in Equation (19) can be applied to the microscopic spacetime structures of quantum particles and, furthermore, in order to specify a particular form for the quantity $g^{00}$, we will adopt Weyl postulate, even though the postulate has mainly been used for considerations of macroscopic phenomena. Weyl postulate requires that the geodesics of the perfect fluid particles are orthogonal to a family of spacelike hypersurfaces. As a consequence, a commoving frame can be introduced such that the line element can be written in the form [10]

$$
d s^{2}=g_{00} c^{2} d t^{2}-a^{2}(t) g_{\alpha \beta} d x^{\alpha} d x{ }^{\beta} .
$$

It is noted that Weyl hypothesis allows us to think of the geometry in which spatial structures evolve over time. With this view, quantum particles that are formed from the microscopic spacetime structures can be regarded as three-dimensional differentiable manifolds which can be identified with normal elementary particles in threedimensional Euclidean space. As a consequence, we will assume that the matter density $\rho$ in Equation (32) also satisfies the Poisson equation for a potential $V$ in classical physics

$$
\nabla^{2} V=4 \pi k_{2} \rho
$$

where $k_{2}$ is a dimensional constant. Normally, Poisson equation is used to describe the potential field of a conservative force, which is time-independent. However, Poisson equation can also be used for time-dependent potentials if the Coulomb gauge is applied. In fact, Poisson equation can also be formulated for non-conservative forces in which the potentials are time-dependent [16]. And, even though it conserves the energy-momentum tensor, general relativity is non-conservative. Therefore, we can assume that the potential in Poisson equation given in Equation (34) is time-dependent. As in the case of Einstein theory of general relativity in which the field equations are proposed by observing the similarity between the Bianchi identities $\nabla_{\beta}\left(R^{\alpha \beta}-\frac{1}{2} g^{\alpha \beta} R\right) \equiv 0$ and the conservation of the energy-momentum tensor $\nabla_{\beta} T^{\alpha \beta} \equiv 0$, in the following we will assume that the scalar potential $V$ and the Ricci scalar to be related by the relation

$$
V=k_{3} R
$$

where $k_{3}$ is an undetermined dimensional constant. With the above assumptions, from Equations (32), (34) and (35) we obtain

$$
\nabla^{2}\left(k_{3} R\right)=\frac{4 \pi k_{2} k_{1}}{2 c} g^{00} \partial_{t} R
$$

Equation (36) is rewritten as

$$
\partial_{t} R=\frac{c k_{3}}{2 \pi k_{1} k_{2} g^{00}} \nabla^{2} R .
$$

In order to investigate further we need to specify the time component $g^{00}$ of the metric tensor $g^{\alpha \beta}$. It is seen from Equations (2) and (4) given in Appendix 3 that a real spacetime structure that is described by the Ricci tensor can admit a real metric tensor or an imaginary metric tensor. If the metric tensor $g^{\alpha \beta}$ is real then we obtain a diffusion equation

$$
\partial_{t} R=k \nabla^{2} R
$$

where the constant $k=c k_{3} /\left(2 \pi k_{1} k_{2} g^{00}\right)$. While investigating the theory of the Brownian movement of particles suspended in a liquid, Einstein derived the following one-dimensional differential equation for diffusion [17]

$$
\frac{\partial f(x, t)}{\partial t}=D \frac{\partial^{2} f(x, t)}{\partial x^{2}}
$$

where $f(x, t)$ can be identified with the concentration per unit length of the number of particles or of the substance under study, and $D$ is the coefficient of diffusion. The solution to Equation (39) is 


$$
f(x, t)=\frac{M}{\sqrt{4 \pi D t}} e^{-\frac{x^{2}}{4 D t}}
$$

where $M=\int_{-\infty}^{\infty} f(x, t) d x$ is the total number of particles or the total mass of the substance. For the case of a threedimensional diffusion equation given in Equation (38), solutions can be found to take the form [18]

$$
R(x, y, z, t)=\frac{M}{(\sqrt{4 \pi k t})^{3}} e^{-\frac{x^{2}+y^{2}+z^{2}}{4 k t}} .
$$

Equation (41) determines the probabilistic distribution of an amount of geometrical substance $M$ which is defined via the Ricci scalar $R$ and manifests as observable matter. On the other hand, if the metric tensor $g^{\alpha \beta}$ is imaginary, then since the Ricci tensor $R_{\alpha \beta}$ is real and the Ricci scalar $R$ is a contraction of the metric tensor and the Ricci tensor given by the relation $R=g^{\alpha \beta} R_{\alpha \beta}$, the Ricci scalar $R$ is imaginary. If we let $R=i \Psi$ then Equation (38) can be written as

$$
\partial_{t} \Psi=i k \nabla^{2} \Psi
$$

Equation (42) is similar to the free particle Schrödinger wave equation in quantum mechanics

$$
\frac{\partial \psi(\mathbf{r}, \mathbf{t})}{\partial t}=i \frac{\hbar}{2 \mu} \nabla^{2} \psi(\mathbf{r}, \mathbf{t}) .
$$

The similarity between Equations (40) and (41) suggests that the Schrödinger wavefunction $\psi(\mathbf{r}, \mathbf{t})$ may intrinsically be related to the geometrical structure of spacetime that can be materialised to become observable as quantum particles. However, it is seen that unless $\partial_{t} R=0$, Equation (42) can only be realised within the existing framework of mathematics if there exist real functions whose rates of change are imaginary functions. In fact, such functions can be used to describe real physical processes without their property of producing imaginary rates being realised. For example, if the rates of change of a real function $f(x)$ are given as $d f(x) / d x=$ $i k_{1} f(x)$ and $d(d f(x) / d x) / d x=i k_{2} d f(x) / d x$, where $k_{1}$ and $k_{2}$ are real, then $d^{2} f(x) / d x^{2}+k_{1} k_{2} f(x)=0$ is a real equation, which can be used to describe a wave motion. If we generalise Equation (38) by assuming that the Ricci scalar R can take complex-valued values then a complex solution to Equation (38) can be obtained as

$$
\mathrm{R}(x, y, z, t)=\frac{M}{(\sqrt{4 \pi i k t})^{3}} e^{i\left(\frac{x^{2}+y^{2}+z^{2}}{4 k t}\right)} .
$$

Equation (44) can also be rewritten in the form

$$
\begin{aligned}
& \mathrm{R}(x, y, z, t)=\frac{-M}{(\sqrt{4 \pi k t})^{3}}\left(\frac{\sqrt{2}+i \sqrt{2}}{2}\right) \\
& \times\left(\cos \left(\frac{x^{2}+y^{2}+z^{2}}{4 k t}\right)+i \sin \left(\frac{x^{2}+y^{2}+z^{2}}{4 k t}\right)\right) .
\end{aligned}
$$

\section{Spacetime Structures of Elementary Particles}

In this section we investigate spacetime structures of quantum particles by deriving equations that can be used to construct line elements for given Ricci scalar curvatures. The diffusion equation given in Equation (38) describes the density fluctuations of a geometrical substance that is undergoing diffusion. We assume that the geometrical substance materialises to appear as quantum particles from spacetime structures. For the Ricci scalar given in Equation (41), due to the spatial symmetry of the Ricci scalar, we seek a line element of the form

$$
d s^{2}=D(c d t)^{2}-A(x, y, z, t)\left[(d x)^{2}+(d y)^{2}+(d x)^{2}\right]
$$

where $D$ is constant. As shown in Appendix 3, the quantity $A(x, y, z, t)$ satisfies the following differential equation

$$
\begin{aligned}
& -\frac{3}{c^{2} D A} \frac{\partial^{2} A}{\partial t^{2}}+\frac{2}{A^{2}} \nabla^{2} A+\frac{3}{2 A^{3}}(\nabla A)^{2} \\
& =\frac{M}{(\sqrt{4 \pi k t})^{3}} e^{-\frac{x^{2}+y^{2}+z^{2}}{4 k t}} .
\end{aligned}
$$

Asymptotically, Equation (47) describes a wave motion for $t \rightarrow \infty$ given the gauge condition that involves the first derivatives. Those quantum particles that can be described by Equation (47) are short-lived subatomic particles. They appear for a short time and then disappear into the purely gravitational field with a wave motion when $\mathrm{R} \rightarrow 0$. However, whether Equation (47) can be solved to obtain exact solutions requires further investigation. If the metric tensor of the line element given in Equation (46) is complex, then instead of Equation (47), we obtain the following equation for the complex function $A(x, y, z, t)$

$$
\begin{aligned}
& -\frac{3}{c^{2} D A} \frac{\partial^{2} A}{\partial t^{2}}+\frac{2}{A^{2}} \nabla^{2} A+\frac{3}{2 A^{3}}(\nabla A)^{2} \\
& =\frac{M}{(\sqrt{4 \pi i k t})^{3}} e^{i\left(\frac{x^{2}+y^{2}+z^{2}}{4 k t}\right)} .
\end{aligned}
$$

Now, it is noted that if the Ricci scalar curvature is time-independent then Equation (38) reduces to Laplace equation

$$
\nabla^{2} \mathrm{R}=0
$$

From the relation $j^{\alpha}=\left(\rho, \mathbf{j}_{i}\right)=\frac{1}{2} g^{\alpha \beta} \nabla_{\beta} R=\frac{1}{2} g^{\alpha \beta} \partial_{\beta} R$, for the case of a symmetric metric tensor, we have $\rho=0$, therefore the spatial structure of a quantum particle is visualised only in terms of the three-current $\mathbf{j}_{i}$. Apart from the interesting question that arises from this result about what form of matter the three-current $\mathbf{j}_{i}$ to represent, such physical structure is possible only when the metric tensor itself depends explicitly on time but the contraction $R=g^{\alpha \beta} R_{\alpha \beta}$ reduces the Ricci scalar to time-independent. 
For example, from the defined relation of the Ricci scalar curvature as $R=g^{00} R_{00}+g^{11} R_{11}+g^{22} R_{22}+g^{33} R_{33}$, if $g^{00} R_{00}$ is time-independent and $g^{11}, g^{11}$ and $g^{11}$ are time-dependent, a time-independent Ricci scalar can be obtained if $g^{11} R_{11}+g^{22} R_{22}+g^{33} R_{33} \equiv 0$.

If the spatial structure of a quantum particle is considered to be spherically symmetric then its materialised spatial structure can be described using spherical coordinates $(r, \theta, \phi)$. In this case Equation (49) takes the form

$$
\begin{aligned}
& \frac{1}{r^{2}} \frac{\partial}{\partial r}\left(r^{2} \frac{\partial \mathrm{R}}{\partial r}\right)+\frac{1}{r^{2} \sin \theta} \frac{\partial}{\partial \theta}\left(\sin \theta \frac{\partial \mathrm{R}}{\partial \theta}\right) \\
& +\frac{1}{r^{2} \sin ^{2} \theta}\left(\frac{\partial^{2} \mathrm{R}}{\partial \phi^{2}}\right)=0 .
\end{aligned}
$$

The general solution for the Ricci scalar curvature R can be found as

$$
\mathrm{R}(r, \theta, \phi)=\sum_{l=0}^{\infty} \sum_{m=-l}^{l}\left(A_{l m} r^{l}+B_{l m} r^{-(l+1)}\right) \mathrm{Y}_{l m}(\theta, \phi)
$$

where $\mathrm{Y}_{l m}(\theta, \phi)$ is the spherical harmonics and the coefficients $A_{l m}$ and $B_{l m}$ can be determined from the boundary conditions [19].

If the spatial structure of an elementary particle is considered to be cylindrically symmetric, such as a thin disc that forms the rotor of a gyroscope, then its materialised spatial structure can be described using cylindrical coordinates $(\rho, \phi, z)$. The Laplace equation given in Equation (49) now takes the form

$$
\frac{\partial^{2} \mathrm{R}}{\partial \rho^{2}}+\frac{1}{\rho} \frac{\partial \mathrm{R}}{\partial \rho}+\frac{1}{\rho^{2}} \frac{\partial^{2} \mathrm{R}}{\partial \phi^{2}}+\frac{\partial^{2} \mathrm{R}}{\partial \mathrm{z}^{2}}=0 .
$$

The general form of the solution for the boundary problem where the cylinder has a radius $a$ and a height $L$ is found as

$$
\begin{aligned}
& \mathrm{R}(\rho, \phi, z) \\
& =\sum_{m=0}^{\infty} \sum_{n=1}^{\infty} J_{m}\left(k_{m n} \rho\right) \sinh \left(k_{m n} z\right)\left(\begin{array}{l}
A_{m n} \sin (m \phi) \\
+B_{m n} \cos (m \phi)
\end{array}\right)
\end{aligned}
$$

where $J_{m}(x)$ is Bessel function, $k_{m n}=x_{m n} / a$ with $x_{m n}$ are the roots of $J_{m}\left(x_{m n}\right)=0$, and the coefficients $A_{m n}$ and $B_{m n}$ can be determined from the boundary conditions [19].

Although it is almost impossible to construct line elements for the whole value of the Ricci scalar curvatures given in Equations (51) and (53), it is possible to construct a line element for each quantum state for discrete values of $l$ and $m$. For example, the quantum state with $l=0$ and $m=0$ for the spherically symmetric Ricci scalar curvature given in Equation (51) is

$$
\mathrm{R}(r)=\frac{1}{\sqrt{4 \pi}}\left(\frac{B_{00}}{r}+A_{00}\right) .
$$

If we assume a spherically symmetric line element of the following form

$$
d s^{2}=D(c d t)^{2}-A(r)(d r)^{2}-r^{2}(d \theta)^{2}-r^{2} \sin ^{2} \phi(d \phi)^{2}
$$

where $D, A_{00}$ and $B_{00}$ are undetermined constants, then using the result obtained in Appendix 3, we arrive at the differential equation

$$
\frac{r}{2 A^{2}} \frac{d A}{d r}+\frac{2}{r^{2}}\left(1-\frac{1}{A}\right)=\frac{1}{\sqrt{4 \pi}}\left(\frac{B_{00}}{r}+A_{00}\right) .
$$

Equation (56) is a first order non-linear differential equation and it can be shown that in general there exists a unique solution to the initial value problem that involves such equation.

\section{A Relationship between Schrödinger Wavefunctions and Spacetime Structures}

In this section, we show that there is a relationship between Schrödinger wavefunctions and the spacetime structures of a quantum system in the sense that Schrödinger wavefunctions are considered purely as mathematical objects that can be used for the construction of spacetime structures of the quantum states of a quantum system. In order to construct the spacetime structures for quantum particles, we observed the similarity between the equation $\rho=\left(k_{1} / 2 c\right) g^{00} \partial_{t} R$ and the equation $4 \pi k_{2} \rho=$ $\nabla^{2} V$. We assumed that the scalar potential $\varphi$ and the Ricci scalar curvature $R$ to be related by the relation $V=k_{3} R$, where $k_{1}, k_{2}$ and $k_{3}$ are undetermined dimensional constants. In the following we will discuss a procedure to construct spacetime structures for the quantum states of a quantum system in which Schrödinger wavefunctions are employed as a pathway. We also assume that the relation $V=k_{3} R$ is hold for any potential defined in classical physics. Since Schrödinger's original works were on the time-independent quantum states of the hydrogen atom, we first recapture the main ideas of Schrödinger's method to obtain the time-independent wave equation for the hydrogen atom [20,21]. Schrödinger commenced with the Hamilton-Jacobi equation, written in terms of the Cartesian coordinates $(x, y, z)$ as

$$
\left(\frac{\partial S}{\partial x}\right)^{2}+\left(\frac{\partial S}{\partial x}\right)^{2}+\left(\frac{\partial S}{\partial x}\right)^{2}-2 m\left(E+\frac{k q^{2}}{r}\right)=0 .
$$

However, in order to obtain a partial differential equation that would give rise to the required results, Schrödinger introduced a new function $\psi$, which is real, single-valued and twice differentiable, through the relation

$$
S=\hbar \ln \psi
$$

where the action $S$ is defined by

$$
S=\int L d t
$$

and $L$ is the Lagrangian defined by

$$
L=T-\varphi
$$

with $T$ is the kinetic energy and $\varphi$ is the potential energy. In terms of the new function $\psi$, Equation (57) takes the form 


$$
\left(\frac{\partial \psi}{\partial x}\right)^{2}+\left(\frac{\partial \psi}{\partial x}\right)^{2}+\left(\frac{\partial \psi}{\partial x}\right)^{2}-\frac{2 m}{\hbar^{2}}\left(E+\frac{k q^{2}}{r}\right) \psi^{2}=0
$$

Then, by applying the principle of least action defined in classical dynamics, $\delta \int L d t=0$, Schrödinger arrived at the required equation

$$
\nabla^{2} \psi+\frac{2 m}{\hbar^{2}}\left(E+\frac{k q^{2}}{r}\right) \psi=0
$$

Now we show that Schrödinger wavefunction $\psi$ can be used to construct the spacetime structures of the quantum states of the hydrogen atom. By using the defined relations $L=d S / d t, d S / d t=\partial_{t} S+\sum_{\mu=1}^{3} \partial_{\mu} S\left(d x^{\mu} / d t\right)$, $T=m \sum_{\mu=1}^{3}\left(d x^{\mu} / d t\right)^{2}$ and $\varphi=T-L$, we obtain

$$
\varphi=m \sum_{\mu=1}^{3}\left(d x^{\mu} / d t\right)^{2}-\partial_{t} S+\sum_{\mu=1}^{3} \partial_{\mu} S\left(d x^{\mu} / d t\right) .
$$

In terms of the Schrödinger wavefunction $\psi$, Equation (63) can be rewritten as

$$
\varphi=m \sum_{\mu=1}^{3}\left(d x^{\mu} / d t\right)^{2}-\hbar \frac{\partial_{t} \psi+\sum_{\mu=1}^{3} \partial_{\mu} \psi\left(d x^{\mu} / d t\right)}{\psi}
$$

From the assumed relations $V=k_{3} \mathrm{R}$ and $V=\varphi / m$, we obtain the following relationship between the Schrödinger wavefunction $\psi$ and the Ricci scalar curvature $\mathrm{R}$

$$
\mathrm{R}=\frac{1}{k_{3}}\left(\begin{array}{l}
\sum_{\mu=1}^{3}\left(d x^{\mu} / d t\right)^{2} \\
-\frac{\hbar}{m} \frac{\partial_{t} \psi+\sum_{\mu=1}^{3} \partial_{\mu} \psi\left(d x^{\mu} / d t\right)}{\psi}
\end{array}\right) .
$$

Since we will use spherical coordinates for the Schrödinger wave equation given in Equation (62), the Ricci scalar curvature should also be written in terms of spherical coordinates $(r, \theta, \phi)$. The Ricci scalar curvature given in Equation (65) then takes the form

$$
\mathrm{R}=\frac{1}{k_{3}}\left(\begin{array}{c}
\left(\frac{d r}{d t}\right)^{2}+r^{2} \sin ^{2} \phi\left(\frac{d \theta}{d t}\right)^{2}+r^{2}\left(\frac{d \phi}{d t}\right)^{2} \\
-\frac{\hbar}{m} \frac{\partial_{t} \psi+\partial_{r} \psi \frac{d r}{d t}+\partial_{\theta} \psi \frac{d \theta}{d t}+\partial_{\phi} \psi \frac{d \phi}{d t}}{\psi}
\end{array}\right)
$$

The eigenfunctions $\psi_{n l m}(r, \theta, \phi)$ for the hydrogen atom, which are solutions to the Schrödinger wave equation given in Equation (62), can be found in the form

$$
\psi_{n l m}(r, \theta, \phi)=R_{n l}(r) Y_{l m}(\theta, \phi)
$$

where the spherical harmonics $Y_{l m}(\theta, \phi)$ and the radial functions $R_{n l}(r)$ are given as

$$
Y_{l m}(\theta, \phi)=(-1)^{m}\left(\frac{(2 l+1)(l-m) !}{4 \pi(l+m) !}\right)^{\frac{1}{2}} P_{l}^{m}(\cos \theta) e^{i m \phi}
$$

$$
R_{n l}(r)=-\left(\left(\frac{2}{n a_{0}}\right)^{3} \frac{(n-l-1) !}{2 n((n+l) !)^{3}}\right)^{\frac{1}{2}} e^{-\frac{\rho}{2}} \rho^{l} L_{n+l}^{2 l+1}(\rho)
$$

where $\rho=2 r / n a_{0}$ and $a_{0}=4 \pi \varepsilon_{0} \hbar^{2} / m q^{2}$ [8]. From these solutions, the first few normalised wave functions for the hydrogen atom and their corresponding Ricci scalar curvatures are given below

$$
\begin{aligned}
& \psi_{100}(r, \theta, \phi)=\frac{1}{\sqrt{\pi}}\left(\frac{1}{a_{0}}\right)^{\frac{3}{2}} e^{-\frac{r}{a_{0}}} \\
& \mathrm{R}=\frac{1}{k_{3}}\left(\begin{array}{l}
\left(\frac{d r}{d t}\right)^{2}+r^{2} \sin ^{2} \phi\left(\frac{d \theta}{d t}\right)^{2} \\
+r^{2}\left(\frac{d \phi}{d t}\right)^{2}+\frac{\hbar}{m a_{0}} \frac{d r}{d t}
\end{array}\right) \\
& \psi_{200}(r, \theta, \phi)=\frac{1}{4 \sqrt{2 \pi}}\left(\frac{1}{a_{0}}\right)^{\frac{3}{2}}\left(2-\frac{r}{a_{0}}\right) e^{-\frac{r}{2 a_{0}}} \\
& \mathrm{R}=\frac{1}{k_{3}}\left(\begin{array}{l}
\left(\frac{d r}{d t}\right)^{2}+r^{2} \sin ^{2} \phi\left(\frac{d \theta}{d t}\right)^{2} \\
+r^{2}\left(\frac{d \phi}{d t}\right)^{2}+\frac{3 \hbar}{m a_{0}} \frac{d r}{d t}
\end{array}\right) \\
& \psi_{300}(r, \theta, \phi)=\frac{1}{81 \sqrt{3 \pi}}\left(\frac{1}{a_{0}}\right)^{\frac{3}{2}}\left(27-\frac{18 r}{a_{0}}+\frac{2 r^{2}}{a_{0}^{2}}\right) e^{-\frac{r}{3 a_{0}}} \\
& \mathrm{R}=\frac{1}{k_{3}}\left(\begin{array}{l}
\left(\frac{d r}{d t}\right)^{2}+r^{2} \sin ^{2} \phi\left(\frac{d \theta}{d t}\right)^{2} \\
+r^{2}\left(\frac{d \phi}{d t}\right)^{2}+\frac{\hbar}{m}\left(\frac{54-4 r}{a_{0}}\right) \frac{d r}{d t}
\end{array}\right) .
\end{aligned}
$$

It is seen from the above results that unless particular conditions are imposed, such as the constancy of the kinetic energy of the electron on each quantum state, as in Borh's model, the mathematical construction of possible spacetime structures of the quantum states would require laborious mathematical investigations. However, we would like to give the following interesting discussion about the geometric structures of spacetime that are closely related to the undeterministic characteristics of the quantum states of a quantum system [15]. If we consider the quantum spacetime structures as embedded surfaces in the Euclidean space $\mathbf{R}^{3}$ then the Ricci scalar curvature $\mathrm{R}$ is related to the Gaussian curvature $K=1 / k_{1} k_{2}$ as

$$
\mathrm{R}=\frac{2}{k_{1} k_{2}}
$$

where $k_{1}$ and $k_{2}$ are the principal radii of the surface. Consider a surface defined by the relation $x^{3}=f\left(x^{1}, x^{2}\right)$ in Cartesian coordinates $\left(x^{1}, x^{2}, x^{3}\right)$. The Ricci scalar curvature given in Equation (76) can be found as 


$$
\mathrm{R}=\frac{2\left(f_{11} f_{22}-\left(f_{12}\right)^{2}\right.}{\left(1+f_{1}^{2}+f_{2}^{2}\right)^{2}}
$$

where $f_{\mu}=\partial f / \partial x^{\mu}$ and $f_{\mu v}=\partial^{2} f / \partial x^{\mu} \partial x^{v}$ [22]. Let $P$ be a 3-dimensional physical quantity which plays the role of the momentum $p$ in the 2-dimensional action integral. The quantity $P$ can be identified with the surface density of a physical quantity, such as charge. Since the momentum $p$ is proportional to the curvature $\kappa$, which determines the planar path of a particle, in the 3-dimensional space the quantity $P$ should be proportional to the Ricci scalar curvature $\mathrm{R}$, which is used to characterise a surface. If we consider a surface action integral of the form $S=\int P d A=\int(q / \pi) \operatorname{R} d A$, where $q$ is a universal constant, which plays the role of Planck's constant, then we have

$$
S=\frac{q}{\pi} \int \frac{f_{11} f_{22}-\left(f_{12}\right)^{2}}{\left(1+f_{1}^{2}+f_{2}^{2}\right)^{3 / 2}} d x^{1} d x^{2} .
$$

According to the calculus of variations, in order to extremise the action integral of the form $S=\int L\left(f, f_{\mu}, f_{\mu v}, x^{\mu}\right) d x^{1} d x^{2}$, the functional $L\left(f, f_{\mu}, f_{\mu \nu}, x^{\mu}\right)$ must satisfy the differential equations

$$
\frac{\partial L}{\partial f}-\frac{\partial}{\partial x^{\mu}} \frac{\partial L}{\partial f_{\mu}}+\frac{\partial^{2}}{\partial x^{\mu} \partial x^{v}} \frac{\partial L}{\partial f_{\mu v}}=0
$$

However, it is straightforward to verify that with the functional of the form obtained from Equation (78), $L=(q / 2 \pi)\left(f_{11} f_{22}-\left(f_{12}\right)^{2}\right) /\left(1+f_{1}^{2}+f_{2}^{2}\right)^{3 / 2}$, the differential equation given by Equation (79) is satisfied by any surface. Hence, we can generalise Feynman's postulate to formulate a quantum theory in which the transition amplitude between states of a quantum mechanical system is a sum over random surfaces, provided the functional $P$ in the action integral $S=\int P d A$ is taken to be proportional to the Ricci scalar curvature $\mathrm{R}$ of a surface. Consider a closed surface and assume that we have many such different surfaces which are described by the higher dimensional homotopy groups. As for the case of positive curvatures, we choose from among the homotopy class a representative spherical surface, in which case we can write

$$
\oint P d A=\frac{q}{4 \pi} \oint d \Omega,
$$

where $d \Omega$ is an element of solid angle. Since $\oint d \Omega$ depends on the homotopy class of the sphere that it represents, we have $\oint d \Omega=4 \pi n$, where $n$ is the topological winding number of the higher dimensional homotopy group. From this result we obtain a generalised Bohr quantum condition

$$
\oint P d A=n q .
$$

From the result obtained in Equation (81), as in the case of Bohr's theory of quantum mechanics, we may consider a quantum process in which a physical entity transits from one surface to another with some radiation-like quantum created in the process. Since this kind of physical process can be considered as a transition from one homotopy class to another, the radiation-like quantum may be the result of a change of the topological structure of the physical system, and so it can be regarded as a topological effect. Furthermore, it is interesting to note that the action integral $(q / 2 \pi) \oint \mathrm{R} d A$ is identical to Gauss's law in electrodynamics and the constant $q$ can be identified with the charge of a particle. In this case the charge $q$ represents the topological structure of a physical system, and must exist in multiples of $q$. Hence, the charge of a physical system, such as an elementary particle, may depend on the topological structure of the system and is classified by the homotopy group of closed surfaces. This result may shed some light on why charge is quantised even in classical physics. We would also like to mention here that the physical entity of magnetic monopole that was introduced by Dirac can also be formulated in terms of topology and the homotopy group as discussed above for the electric charge if instead of one-dimensional we introduce a three-dimensional temporal manifold. In particular, we also show that the Dirac relationship $\hbar c / q_{e} q_{m}=2$ between the electric charge $q_{e}$ and the magnetic charge $q_{m}$ can be obtained from topological characteristics and invariants [5].

\section{Conclusion}

We have shown in this work that physical formulations in classical physics that include Newton's second law of motion, the field equations of the electromagnetic field and the field equations of the gravitational field can be formulated in a general covariant form so that the formulations differ only by the nature of the geometrical objects that represent the corresponding physical entities. In particular, when the field equations of the gravitational field are formulated covariantly using Bianchi identities then instead of viewing quantum particles as mass points we show that it is possible to represent them as threedimensional differentiable manifolds which possess geometric and topological structures, and these structures can be determined by Schrödinger wavefunctions by identifying the classical potential with the Ricci scalar curvature, and therefore we can extend the description of the dynamics of quantum particles in the current formulation of physics as mass points to the dynamics of elementary particles as three-dimensional differentiable manifolds in an ambient space. Being viewed as threedimensional differentiable manifolds, quantum particles may possess internal geometric symmetries that give rise to intrinsic dynamics. If elementary particles are assumed to remain as stable geometric structures then their intrinsic dynamics should be described by continuous isometric transformations, which is an isometric embedding into the spatiotemporal manifold [23]. We can describe the evolution of quantum particles as a change of their geometric structures through evolutionary processes, such as the Ricci flow or the field equations of general relativity, rather than their motion in an ambient space as mass points. For a purely geometrical formulation of physical theories we may speculate that the continuous isometric embedding of three-dimensional Riemannian 
manifolds should be viewed as a continuous process of materialising spacetime structures rather than the motion of a solid physical object through space with respect to time. It is also possible to suggest that the process of materialisation from spacetime structures under a continuous isometric embedding will decompose the geometric structure of spatiotemporal manifold in the forms of $n$-cells which in turns will give rise to physical effects which manifest as physical fields, such as the gravitational field or the electromagnetic field. Therefore, mathematically, we consider quantum particles as three-dimensional differentiable manifolds which may have intrinsic geometric and topological structures of $\mathrm{CW}$ complexes.

As a further remark, we would like to mention here that even though our discussions have been focused on the quantum objects, the results are equally applied to macroscopic phenomena.

\section{Acknowledgements}

I would like to thank the reviewers for their suggestions for the improvement of the paper. I would also like to thank the administration of SciEP for their advices for the preparation for the publication of this paper.

\section{Appendix 1}

In differential geometry, the covariant derivative of a contravariant tensor of second rank $A^{\alpha \beta}$ is given by

$$
\nabla_{\gamma} A^{\alpha \beta}=\partial_{\gamma} A^{\alpha \beta}+\Gamma_{\sigma \gamma}^{\alpha} A^{\sigma \beta}+\Gamma_{\sigma \gamma}^{\alpha} A^{\alpha \sigma}
$$

The partial time derivative of Equation (1) is

$$
\begin{aligned}
& \partial_{t}\left(\nabla_{\gamma} A^{\alpha \beta}\right) \\
& =\partial_{t}\left(\partial_{\gamma} A^{\alpha \beta}\right)+\left(\partial_{t} \Gamma_{\sigma \gamma}^{\alpha}\right) A^{\sigma \beta}+\Gamma_{\sigma \gamma}^{\alpha}\left(\partial_{t} A^{\sigma \beta}\right) \\
& +\left(\partial_{t} \Gamma_{\sigma \gamma}^{\alpha}\right) A^{\alpha \sigma}+\Gamma_{\sigma \gamma}^{\alpha}\left(\partial_{t} A^{\alpha \sigma}\right) .
\end{aligned}
$$

Under the coordinate transformation $x^{\prime \alpha}=f^{\alpha}\left(x^{\beta}\right)$, the tensor $A^{\alpha \beta}$ is transformed as

$$
A^{\prime \alpha \beta}=\frac{\partial x^{\prime \alpha}}{\partial x^{\rho}} \frac{\partial x^{\prime} \beta}{\partial x^{\sigma}} A^{\rho \sigma} .
$$

If the coordinate transformation is time-independent then the partial time derivative of the tensor $A^{\alpha \beta}$ is also a tensor which is transformed according to the rule

$$
\frac{\partial A^{\prime \alpha \beta}}{\partial t}=\frac{\partial x^{\prime \alpha}}{\partial x^{\rho}} \frac{\partial x^{\prime} \beta}{\partial x^{\sigma}} \frac{\partial A^{\rho \sigma}}{\partial t} .
$$

In this case, we have

$$
\begin{aligned}
& \nabla_{\gamma}\left(\partial_{t} A^{\alpha \beta}\right) \\
& =\partial_{\gamma}\left(\partial_{t} A^{\alpha \beta}\right)+\Gamma_{\sigma \gamma}^{\alpha}\left(\partial_{t} A^{\sigma \beta}\right)+\Gamma_{\sigma \gamma}^{\alpha}\left(\partial_{t} A^{\alpha \sigma}\right) .
\end{aligned}
$$

It is observed from Equations (2) and (5) that if we impose the following condition on Equation (2)

$$
\left(\partial_{t} \Gamma_{\sigma \gamma}^{\alpha}\right) A^{\sigma \beta}+\left(\partial_{t} \Gamma_{\sigma \gamma}^{\alpha}\right) A^{\alpha \sigma}=0
$$

then we obtain the identity

$$
\nabla_{\gamma}\left(\partial_{t} A^{\alpha \beta}\right)=\partial_{t}\left(\nabla_{\gamma} A^{\alpha \beta}\right)
$$

For a metric tensor $g^{\alpha \beta}$ we have the identity $\nabla_{\gamma}\left(\partial_{t} g^{\alpha \beta}\right)=\partial_{t}\left(\nabla_{\gamma} g^{\alpha \beta}\right) \equiv 0$, and therefore from the field equations $\nabla_{\beta} R^{\alpha \beta}=0$ we arrive at

$$
\frac{\partial g_{\alpha \beta}}{\partial t}=k R_{\alpha \beta}
$$

where $k$ is a scaling factor.

\section{Appendix 2}

In differential geometry, the position vector $\mathbf{r}(s)$, the unit tangent vector $\mathbf{t}(s)$, the unit principal normal vector $\mathbf{p}(s)$ and the unit binormal vector $\mathbf{b}(s)$, defined by the relation $\mathbf{b}(s)=\mathbf{t}(s) \times \mathbf{p}(s)$, satisfy the Frenet equations [22]

$$
\frac{d \mathbf{t}}{d s}=\kappa \mathbf{p}, \frac{d \mathbf{p}}{d s}=-\kappa \mathbf{t}+\varrho \mathbf{b}, \frac{d \mathbf{b}}{d s}=-\varrho \mathbf{p}
$$

where $\kappa(s)$ and $\varrho(s)$ are the curvature and the torsion respectively, and $d s=\sqrt{d \mathbf{r} \cdot d \mathbf{r}}$ is the linear element. If we consider the motion of a particle in a plane, as in the case of Bohr's model of a hydrogen-like atom, the Frenet equations reduce to

$$
\frac{d \mathbf{t}}{d s}=\kappa \mathbf{p}, \frac{d \mathbf{p}}{d s}=-\kappa \mathbf{t} .
$$

By differentiation, we obtain the following system of differential equations

$$
\begin{aligned}
& \frac{d^{2} \mathbf{t}}{d s^{2}}-\frac{d(\ln \kappa)}{d s} \frac{d \mathbf{t}}{d s}+\kappa^{2} \mathbf{t}=0 \\
& \frac{d^{2} \mathbf{p}}{d s^{2}}-\frac{d(\ln \kappa)}{d s} \frac{d \mathbf{p}}{d s}+\kappa^{2} \mathbf{p}=0 .
\end{aligned}
$$

If the curvature $\kappa(s)$ is assumed to vary slowly along the curve $\mathbf{r}(s)$, so that the condition $d(\ln \kappa) / d s=0$ can be imposed, then $\mathbf{t}(s)$ and $\mathbf{p}(s)$ may be regarded as being oscillating with a spatial period, or wavelength, $\lambda$, whose relationship to the curvature $\kappa$ is found as

$$
\kappa=\frac{2 \pi}{\lambda} \text {. }
$$

In the case of the Bohr's planar model of a hydrogen-like atom with circular orbits, the condition $d(\ln \kappa) / d s=0$ is always satisfied, since the curvature remains constant for each of the orbits. In order to incorporate this elementary differential geometry into quantum mechanics, we identify the wavelength defined in Equation (5) with the de Broglie's wavelength of a particle. This seems to be a natural identification since the spatial period $\lambda$ is the wavelength of the unit tangent vector $\mathbf{t}(s)$. With this assumption, the momentum $p$ of the particle and the curvature $\kappa$ are related through the relation 


$$
p=\hbar \kappa
$$

\section{Appendix 3}

In this appendix, we show in detail the derivation of the equations to determine the metric tensor of the line element given in Equations (46) and (55) in section 3. In differential geometry, the Riemann curvature tensor $R^{\alpha}{ }_{\mu \beta \nu}$ is defined in terms of the affine connection $\Gamma_{\alpha \beta}^{\gamma}$ as

$$
R_{\mu \beta v}^{\alpha}=\frac{\partial \Gamma_{\mu \beta}^{\alpha}}{\partial x^{v}}-\frac{\partial \Gamma_{\mu v}^{\alpha}}{\partial x^{\beta}}+\Gamma_{\mu \beta}^{\lambda} \Gamma_{\lambda v}^{\alpha}-\Gamma_{\mu v}^{\lambda} \Gamma_{\lambda \beta}^{\alpha} .
$$

The contraction of the Riemann curvature tensor given in Equation (1) with respect to the indices $\alpha$ and $\beta$ gives the Ricci tensor

$$
R_{\mu v}=\frac{\partial \Gamma_{\mu v}^{\sigma}}{\partial x^{\sigma}}-\frac{\partial \Gamma_{\mu \sigma}^{\sigma}}{\partial x^{v}}+\Gamma_{\mu \nu}^{\lambda} \Gamma_{\lambda \sigma}^{\sigma}-\Gamma_{\mu \sigma}^{\lambda} \Gamma_{\lambda v}^{\sigma} .
$$

On the other hand, the contraction of the Riemann curvature tensor with respect to the indices $\alpha$ and $\mu$ gives the segmental curvature tensor [24]

$$
Q_{\alpha \beta}=\frac{\partial \Gamma_{\lambda \beta}^{\lambda}}{\partial x^{\alpha}}-\frac{\partial \Gamma_{\lambda \alpha}^{\lambda}}{\partial x^{\beta}} .
$$

It is seen from Equation (3) that if the affine connection $\Gamma_{\alpha \beta}^{\gamma}$ is symmetric with respect to the indices $\alpha$ and $\beta$ then the segmental curvature tensor $Q_{\alpha \beta}$ is anti-symmetric and in this case it might be used to formulate the electromagnetic field as given in Equation (10). In order to formulate the field equations for the gravitational field it is necessary to introduce a symmetric metric tensor $g_{\alpha \beta}$ in terms of which the affine connection $\Gamma_{\alpha \beta}^{\gamma}$ is defined as

$$
\Gamma_{\mu \nu}^{\lambda}=\frac{1}{2} g^{\lambda \sigma}\left(\frac{\partial g_{\sigma v}}{\partial x^{\mu}}+\frac{\partial g_{\sigma \mu}}{\partial x^{v}}-\frac{\partial g_{\mu v}}{\partial x^{\sigma}}\right) .
$$

However, with the introduction of the symmetric metric tensor, it can be shown that the segmental curvature tensor vanishes. This result shows that the electromagnetic field and the gravitational field may not be formulated on the same geometric structure of a Riemannian manifold. With the line element given in Equation (46) in section 3, we obtain the following non-zero components of the affine connection [25]

$$
\begin{gathered}
\Gamma_{01}^{1}=\Gamma_{10}^{1}=\frac{1}{2 c A} \frac{\partial A}{\partial t}, \Gamma_{02}^{2}=\Gamma_{20}^{2}=\frac{1}{2 c A} \frac{\partial A}{\partial t} \\
\Gamma_{03}^{3}=\Gamma_{30}^{3}=\frac{1}{2 c A} \frac{\partial A}{\partial t}, \Gamma_{11}^{0}=\frac{1}{2 c D} \frac{\partial A}{\partial t} \\
\Gamma_{11}^{1}=\frac{1}{2 A} \frac{\partial A}{\partial x}, \Gamma_{11}^{2}=-\frac{1}{2 A} \frac{\partial A}{\partial y}, \Gamma_{11}^{3}=-\frac{1}{2 A} \frac{\partial A}{\partial z} \\
\Gamma_{12}^{1}=\Gamma_{21}^{1}=\frac{1}{2 A} \frac{\partial A}{\partial y}, \Gamma_{12}^{2}=\Gamma_{21}^{2}=\frac{1}{2 A} \frac{\partial A}{\partial x} \\
\Gamma_{13}^{1}=\Gamma_{31}^{1}=\frac{1}{2 A} \frac{\partial A}{\partial z}, \Gamma_{13}^{3}=\Gamma_{31}^{3}=\frac{1}{2 A} \frac{\partial A}{\partial x}
\end{gathered}
$$

$$
\begin{gathered}
\Gamma_{22}^{0}=\frac{1}{2 c D} \frac{\partial A}{\partial t}, \Gamma_{22}^{1}=\frac{1}{2 A} \frac{\partial A}{\partial x}, \Gamma_{22}^{2}=\frac{1}{2 A} \frac{\partial A}{\partial y} \\
\Gamma_{22}^{3}=-\frac{1}{2 A} \frac{\partial A}{\partial z}, \Gamma_{33}^{0}=\frac{1}{2 c D} \frac{\partial A}{\partial t}, \Gamma_{33}^{1}=-\frac{1}{2 A} \frac{\partial A}{\partial x} \\
\Gamma_{33}^{2}=-\frac{1}{2 A} \frac{\partial A}{\partial y}, \Gamma_{33}^{3}=\frac{1}{2 A} \frac{\partial A}{\partial z}, \\
\Gamma_{23}^{2}=\Gamma_{32}^{2}=\frac{1}{2 A} \frac{\partial A}{\partial z}, \Gamma_{23}^{3}=\Gamma_{32}^{3}=\frac{1}{2 A} \frac{\partial A}{\partial y} .
\end{gathered}
$$

From the components of the affine connection given in Equation (5), we obtain

$$
\begin{aligned}
& R_{11}=\frac{1}{2 c^{2} D} \frac{\partial^{2} A}{\partial t^{2}}-\frac{1}{A} \frac{\partial^{2} A}{\partial x^{2}}-\frac{1}{2 A} \frac{\partial^{2} A}{\partial y^{2}} \\
& -\frac{1}{2 A} \frac{\partial^{2} A}{\partial z^{2}}+\frac{3}{4 c^{2} A D}\left(\frac{\partial A}{\partial t}\right)^{2} \\
& +\frac{1}{A^{2}}\left(\frac{\partial A}{\partial x}\right)^{2}+\frac{1}{4 A^{2}}\left(\frac{\partial A}{\partial y}\right)^{2}+\frac{1}{4 A^{2}}\left(\frac{\partial A}{\partial z}\right)^{2} \\
& R_{22}=\frac{1}{2 c^{2} D} \frac{\partial^{2} A}{\partial t^{2}}-\frac{1}{2 A} \frac{\partial^{2} A}{\partial x^{2}}-\frac{1}{A} \frac{\partial^{2} A}{\partial y^{2}} \\
& -\frac{1}{2 A} \frac{\partial^{2} A}{\partial z^{2}}+\frac{3}{4 c^{2} A D}\left(\frac{\partial A}{\partial t}\right)^{2} \\
& +\frac{1}{4 A^{2}}\left(\frac{\partial A}{\partial x}\right)^{2}+\frac{1}{A^{2}}\left(\frac{\partial A}{\partial y}\right)^{2}+\frac{1}{4 A^{2}}\left(\frac{\partial A}{\partial z}\right)^{2} \\
& R_{33}=\frac{1}{2 c^{2} D} \frac{\partial^{2} A}{\partial t^{2}}-\frac{1}{2 A} \frac{\partial^{2} A}{\partial x^{2}}-\frac{1}{2 A} \frac{\partial^{2} A}{\partial y^{2}} \\
& +\frac{1}{A} \frac{\partial^{2} A}{\partial z^{2}}+\frac{3}{4 c^{2} A D}\left(\frac{\partial A}{\partial t}\right)^{2} \\
& R_{00}=-\frac{\partial A}{2 c^{2} A} \frac{\partial^{2} A}{\partial t^{2}}+\frac{1}{4 c^{2} A^{2}}\left(\frac{\partial A}{\partial y}\right)^{2}+\frac{1}{A^{2}}\left(\frac{\partial A}{\partial z}\right)^{2} \\
& +\frac{\partial A}{3}
\end{aligned}
$$

Using $\quad R=g^{00} R_{00}+g^{11} R_{11}+g^{22} R_{22}+g^{33} R_{33}$ we obtain

$$
R=-\frac{3}{c^{2} D A} \frac{\partial^{2} A}{\partial t^{2}}+\frac{2}{A^{2}} \nabla^{2} A+\frac{3}{2 A^{3}}(\nabla A)^{2} .
$$

With the line element given in Equation (55), we obtain the following non-zero components of the affine connection are [26]

$$
\begin{gathered}
\Gamma_{11}^{1}=\frac{1}{2 A} \frac{d A}{d r}, \Gamma_{22}^{1}=-\frac{r}{A}, \Gamma_{12}^{2}=\Gamma_{12}^{2}=\frac{1}{r} \\
\Gamma_{13}^{3}=\Gamma_{31}^{3}=\frac{1}{r}, \Gamma_{33}^{1}=-\frac{r}{A} \sin ^{2} \theta \\
\Gamma_{33}^{2}=-\cos \theta \sin \theta, \Gamma_{23}^{3}=\Gamma_{32}^{3}=\cot \theta .
\end{gathered}
$$


From the components of the affine connection given in Equation (8), we obtain

$$
\begin{aligned}
& R_{11}=-\frac{1}{r A} \frac{d A}{d r}, R_{22}=\frac{1}{A}-\frac{r}{2 A^{2}} \frac{d A}{d r}-1 \\
& R_{33}=\left(\frac{1}{A}-\frac{r}{2 A^{2}} \frac{d A}{d r}-1\right) \sin ^{2} \theta, R_{00}=0 .
\end{aligned}
$$

Using $\quad R=g^{00} R_{00}+g^{11} R_{11}+g^{22} R_{22}+g^{33} R_{33} \quad$ we obtain

$$
\mathrm{R}=\frac{r}{2 A^{2}} \frac{d A}{d r}+\frac{2}{r^{2}}\left(1-\frac{1}{A}\right)
$$

\section{References}

[1] A. Einstein, The Principle of Relativity (Dover Publications, New York, 1952).

[2] Richard S. Hamilton, Three-Manifolds with Positive Ricci Curvature, J. Diff. Geo., 17 (1982) 255-306.

[3] Huai-Dong Cao and Xi-Ping Zhu, A Complete Proof of the Poincaré and Geometrization Conjectures-Application of the Hamilton-Perelman Theory of the Ricci Flow, Asian J. Math., Vol 10, No. 2, 165-492, June 2006.

[4] Vu B Ho, A Classification of Geometric Interactions (Preprint, ResearchGate, 2018), viXra 1805.0329v1.

[5] Vu B Ho, On Dirac Negative Mass and Magnetic Monopole (Preprint, ResearchGate, 2018), viXra 1806.0319v1.

[6] Vu B Ho, Temporal Geometric Interactions (Preprint, Research Gate, 2018), vi Xra 1807.0134v1.

[7] H. Goldstein, Classical Mechanics (Addison-Wesley Inc., Sydney, 1980).
[8] L. D. Landau and E. M. Lifshitz, The Classical Theory of Fields (Pergamon Press, Sydney, 1987).

[9] A. O. Barut, Electrodynamics and Classical theory of Fields and Particles (Dover Publications, Inc., New York, 1980).

[10] Ray D’Inverno, Introducing Einstein's Relativity (Clarendon Press, Oxford, 1992).

[11] D. Kramer, H. Stephanie, E. Hert and M. MacCallum, Exact solutions of Einstein's field equations (Cambridge University Press, 1980).

[12] N. Bohr, Phil. Mag. 26, 1 (1913).

[13] C. Lanczos, The Variational Principles of Mechanics (Dover Publications, New York, 1970).

[14] R. P. Feynman, Rev. Mod. Phys. 20, 367 (1948).

[15] Vu B Ho, On the Principle of Least Action, ResearchGate (2016), viXra 1708.0226v2, Int. J. Phys., 6, 2 (2018)

[16] M.A.L. Marques et al., Time-Dependent Density Functional Theory, Lecture Notes in Physics 706 (Springer, Berlin Heidelberg, 2006).

[17] A. Einstein, Investigations on the theory of the Brownian movement, edited with notes by R. Furth and translated by A. D. Cowper (Dover Publications, Inc., New York, 1956).

[18] Walter A. Strauss, Partial Differential Equation (John Wiley \& Sons, Inc., New York, 1992).

[19] J. D. Jackson, Classical Electrodynamics (John Wiley \& Sons, New York, 1975).

[20] Erwin Schrödinger, Collected Papers on Wave Mechanics (AMS Chelsea Publishing, New York, 1982).

[21] W. Yourgrau and S. Mandelstam, Variational Principles in Dynamics and Quantum Theory (Dover Publications, New York, 1979).

[22] E. Kreyszig, Introduction to Differential Geometry and Riemannian Geometry (University of Toronto Press, 1975)

[23] $\mathrm{Vu} \mathrm{B} \mathrm{Ho}$, On the motion of quantum particles and Euclidean relativity (Preprint, ResearchGate, 2017), viXra 1710.0253v1.

[24] Nikodem J. Poplawski, Spacetime and fields, arXiv:0911.0334v1 [gr-qc] 2 Nov 2009.

[25] Richard C. Tolman, Relativity Thermodynamics and Cosmology (Dover Publications, New York, 1987).

[26] Gary Oas, EPGY Summer Institute SRGR. 